\title{
Connatal Pelizaeus-Merzbacher Disease: A Great Masquerader
}

Sir,

Pelizaeus-Merzbacher disease (PMD) is a rare neurogenetic disorder caused by mutations of the proteolipid protein 1 (PLP1) gene on the X chromosome. Connatal form is characterised by neonatal onset, congenital nystagmus, stridor, hypotonia and diffuse leukoencephalopathy. ${ }^{1}$ We present a case mimicking birth asphyxia, vocal cord palsy, cerebral palsy and neuromuscular disorder.

A male infant was born at $36 \mathrm{wk}$ gestation by normal delivery. Fetal bradycardia was noted prior to birth. He was born pale, hypotonic and needed resuscitation and ventilation at birth. Apgar score was 2 and 4 at 1 and 5 min respectively. He developed bilateral vocal cord palsy and a tracheostomy was performed. Motor developmental milestones were delayed and he had marked hypotonia and hyporeflexia. He also developed ocular nystagmus but was visually very responsive. By 10 mo of age, he made some developmental progress but was still hypotonic with head lag. A diagnosis of "cerebral palsy" was made and was later referred to our centre in view of unusual presentation and hypotonia.

As part of investigations he had normal chromosome analysis, metabolic screening and creatine kinase. Electromyography was suggestive of chronic neurogenic changes. Auditory brain stem responses were abnormal. MRI brain showed diffuse leukodystrophy with a pattern suggestive of Pelizaeus-Merzbacher disease (PMD). Molecular analysis showed the P.G74R (glycine to arginine substitution at codon 74) mutation in PLP1 gene and hence confirmed the diagnosis.

Our case illustrated the difficulties to diagnose PMD. The clinical features are not uncommon in neuromuscular disorder and cerebral palsy. Given adverse perinatal events, abnormal EMG study and neurodevelopmental progression, the possible causes are even extensive. However, diffuse leukodystrophy is characteristic and cranial MRI plays an important role in diagnosis. ${ }^{2}$
Connatal PMD is the most severe form characterised by Leukodystrophy, hypotonia, stridor and nystagmus. Hypotonia later evolves into spasticity that is usually quite severe. It is also associated with seizures, failure to thrive and motor deficits in the long term. These children have limited language skills and usually die before the third decade of life.

PLP1 is the gene located on the long arm of the $X$ chromosome (Xq22) and associated with PMD and Spastic Paraplegia 2. Between $80 \%$ and $95 \%$ of males with PMD have an identifiable alteration in PLP1 which could be duplications (50-70\%), point mutations (20\%) or deletions. ${ }^{3}$ De novo mutations have also been reported. Early diagnosis is important for genetic counselling. Clinician should be aware of a connatal form of PMD simulating cerebral palsy ${ }^{4}$ and spinal muscular atrophy. ${ }^{5}$

Sushil Beri, Nahin Hussain and Jayaprakash A Gosalakkal

Department of Pediatric Neurology, Leicester Royal Infirmary, University Hospitals of Leicester NHS Trust, Leicester, LE1 5WW United Kingdom.

E-mail: sushilberi@gmail.com

[DOI-10.1007/s12098-009-0303-y]

\section{REFERENCES}

1. Garbern JY. Pelizaeus-Merzbacher Disease. eMedicine. Last updated August 2008. http://emedicine.medscape.com/article/ 1153103-overview

2. Ziereisen F, Dan B, Christiaen F et al. Connatal PelizaeusMerzbacher disease in two girls. Paediatric Radiol 2000; 30: 435-438.

3. Garbern J, Cambi F, Shy M et al. The molecular pathogenesis of Pelizaeus-Merzbacher disease. Arch Neurol 1999; 56: 1210-1214.

4. Caord Artal J, Prats Vinas JM, Garaizar Axpe C et al. Congenital Pelizaeus-Merzbacher disease simulating infantile spastic cerebral palsy. Neurologia 1995; 10: 57-58.

5. Kaye EM, Doll RF, Natowicz MR et al. Pelizaeus-Merzbacher disease as spinal muscular atrophy: clinical and molecular studies. Ann Neurol 1994; 36: 916-919. 